Modal Response of Trapezoidal Wing Structures Using Second Order Shape Sensitivities

\author{
Youhua Liu* and Rakesh K. Kapania** \\ Virginia Polytechnic Institute and State University \\ Blacksburg, VA 24061
}

\title{
Introduction
}

The modal response of wing structures is very important for assessing their dynamic response including dynamic aeroelastic instabilities. Moreover, in a recent study ${ }^{1}$ an efficient structural optimization approach was developed using structural modes to represent the static aeroelastic wing response (both displacement and stress).

Sensitivity techniques are frequently used in structural design practices for searching the optimal solutions near a baseline design ${ }^{2,3}$. The design parameters for wing structure include sizing-type variables (skin thickness, spar or rib sectional area etc.), shape variables (the plan surface dimensions and ratios), and topological variables (total spar or rib number, wing topology arrangements etc.). Sensitivities to the shape variables are extremely important because of the nonlinear dependence of stiffness and mass terms on the shape design variables as compared to the linear dependence on the sizing-type design variables.

Kapania and coworkers have obtained the first order shape sensitivities of the modal response, divergence and flutter speed, and divergence dynamic pressure of laminated, box-wing or general trapezoidal built-up wing using various approaches of determining the response sensitivities ${ }^{4-8}$. In this paper, the modal response of general trapezoidal wing structures is approximated using

\footnotetext{
*: Research Assistant, Department of Aerospace and Ocean Engineering, Student member AIAA

**: Professor, Department of Aerospace and Ocean Engineering, Associate Fellow AIAA
} 
shape sensitivities up to the $2^{\text {nd }}$ order. Also different approaches of computing the derivatives are investigated.

\section{Shape Sensitivities}

For a trapezoidal wing, there are four major independent shape variables: 1) sweep angle $\Lambda, 2$ ) aspect ratio $\alpha, 3)$ taper ratio $\tau$, and 4) plan area $A$. All the other dimensions of the wing plate configuration can be calculated using these parameters such as:

$$
s=\sqrt{\alpha A}, a=2 \tau / \alpha(1+\tau), b=2 s / \alpha(1+\tau)
$$

where $s$ is the length of semi-span, $a$ and $b$ are the chord-length at wing tip and root respectively, as shown in Fig. 1.

The sensitivities for the design parameters at a baseline design point indicate trends of variation of the design near the baseline point if the parameters are perturbed. Usually, only the first order derivatives are used. For more accurate results, second order derivatives can used:

$$
\begin{aligned}
& f\left(x^{1}, x^{2}, \cdots, x^{n}\right) \cong f\left(x_{0}^{1}, x_{0}^{2}, \cdots, x_{0}^{n}\right)+\sum_{i=1}^{n}\left(x^{i}-x_{0}^{i}\right) \frac{\partial}{\partial x^{i}} f\left(x_{0}^{1}, x_{0}^{2}, \cdots, x_{0}^{n}\right) \\
& +\frac{1}{2}\left(\sum_{i=1}^{n}\left(x^{i}-x_{0}^{i}\right) \frac{\partial}{\partial x^{i}}\right)^{2} f\left(x_{0}^{1}, x_{0}^{2}, \cdots, x_{0}^{n}\right)
\end{aligned}
$$

\section{Equivalent Plate Analysis (EPA) of Trapezoidal Wing Structures}

In Kapania and $\mathrm{Liu}^{9}$, a general method is presented to analyze trapezoidal, built-up wing structures. The method is based on the Reissner-Mindlin model, a First-order Shear Deformation Theory (FSDT). Free vibration and static response are obtained using the Ritz method. Compared with the methods in Kapania and Lovejoy ${ }^{10}$ and Cortial ${ }^{11}$, the formulation of this method is such that there is no limitation to the wing thickness distribution. As shown in Ref. 9, the method shows a good performance for both static and vibration problems in comparison with the FEA using MSC/NASTRAN. 
Due to its efficiency in determining the natural frequencies and mode shapes of wings, the Equivalent Plate Analysis (EPA) described above can be used to investigate the variation of modal response, that is, to evaluate the shape sensitivities of trapezoidal wing structures under shape changes. For the performances of the baseline design, EPA can still be used, or FEA using a commercial package such as MSC/NASTRAN can be utilized for better accuracy.

\section{Approaches of Shape Variable Sensitivity Evaluation}

There are generally two kinds of methods for the solution of sensitivity derivatives: the finite difference approach and the analytical approach. A finite difference approach is very simple to formulate and implement, but is numerically in-efficient and is sensitive to the step-size used. A too-large step size usually causes significant truncation errors and a too-small step size may lead to large round-off errors. As a result, the more elegant and accurate analytical approach is used as far as it is possible. But for some problems with complex formulation, the derivation of analytical derivatives is too formidable to handle manually, unless Automatic Differentiation (AD) is used ${ }^{12,13}$.

The finite difference approaches can be constructed on the basis of the following formulas:

$$
\begin{aligned}
& f^{\prime}(x)=\frac{f(x+\Delta x)-f(x-\Delta x)}{2 \Delta x}+O\left(\Delta x^{2}\right) \\
& f^{\prime \prime}(x)=\frac{f(x+\Delta x)-2 f(x)+f(x-\Delta x)}{\Delta x^{2}}+O\left(\Delta x^{2}\right)
\end{aligned}
$$

where

$$
\Delta x=\varepsilon \cdot x
$$

in which $\varepsilon$ is the relative step size, and hereafter it is simply called the step size.

The analytical approaches for shape sensitivities of modal response can be based on the following equation

$$
\frac{\partial \lambda_{i}}{\partial \theta}=\left\{\phi_{i}\right\}^{T}\left(\frac{\partial[K]}{\partial \theta}-\lambda_{i} \frac{\partial[M]}{\partial \theta}\right)\left(\phi_{i}\right\}
$$


where 6 is the shape variable, $[K]$ and $[M]$ are the stiffness and mass matrices of the wing structure, $\lambda_{1}$ and $\left\{\phi_{i}\right\}$ are the $i$-th eigenvalue and eigenvetor, and $\left\{\phi_{i}\right\}$ is mass-normalized such that $\left\{\phi_{i}\right\}^{T}[M]\left\{\phi_{i}\right\}=1$. The derivation of Eq. (6) can be found in Ref. 14.

The major difficulty of applying Eqs. (6) lies on the calculation of $\partial[K] / \partial \theta$ and $\partial[M] / \partial \theta$. For instance, consider $\partial[K / / \partial \theta$. According to Ref. 9, the stiffness matrix $[K]$ is formulated as an integral

$$
\left.[K]=\iiint_{V}[C]^{T}[T]^{T}[D][T][C] d V=\int_{-1}^{1} \int_{-1}^{1}[C]^{T}\left(\int_{z_{1}}^{z_{2}}[T]^{T}[D][T]|J| d z\right) C C\right] d \xi d \eta=\int_{-1}^{1} \int_{-1}^{1}[C]^{T}[G][C] d \xi d \eta
$$

where only the inner part $[G]=\int_{z_{1}}^{z_{2}}[T]^{T}[D][T]|J| d z$ is a function of the shape variables, and the Gaussian quadrature is used to obtain the integration on $\xi$ and $\eta$. Therefore,

$$
\frac{\partial[K]}{\partial \theta}=\int_{-1}^{1} \int_{-1}^{1}[C]^{T}\left(\frac{\partial[G]}{\partial \theta}\right)[C] d \xi d \eta
$$

in which ${ }^{\partial G G} / / \partial \theta$ can either be determined analytically or numerically.

Often people make use of the advantages of both the finite difference and analytical approaches in different stages of obtaining some complicated sensitivities. While trying to use the analytical approach as much as possible, in other parts of the process the finite difference is used, as in the case of Ref. 8. This kind of approach is usually called semi-analytical. For the second order sensitivities, there can still be three kinds of approach as specified above. While the formulation for the analytical approach is becoming even more complicated, a scheme as simple as Eq. (4) can be used for the finite difference approach.

\section{Examples and Discussion}

Particulars of the baseline wing structure are as follows: the sweep angle $\Lambda=30^{\circ}$, the aspect ratio $\alpha=3.5$, the taper ratio $\imath=0.5$, the plan area $A=5832 \mathrm{in}^{2}$. There are 4 spars and 10 ribs distributed uniformly under the skins. Details of wing sections, particulars of the skin thickness and dimensions of the spars and ribs can be found in Ref. 9. The wing is clamped at the root. 
The effect of step size on the finite difference approach for sensitivities was investigated for all the four shape variables. The case with the taper ratio is shown in Fig. 2. From all the cases, it is found that for the best results for both the $1^{\text {st }}$ and $2^{\text {nd }}$ order sensitivities, the step size $\varepsilon$ defined in Eq. (5), should be between $0.005 \sim 0.015$ and for fairly accurate results $\varepsilon$ can be between $0.0017 \sim 0.045$.

To evaluate ${ }^{\partial I G} / / \partial \theta$ analytically proved to be formidable except only in some simplified cases. In order to compare the sensitivities using the analytical, semi-analytical and finite difference approaches, a special case of the above baseline wing with a constant thickness distribution was considered so that the analytical derivation of ${ }^{\partial[G} 1 / \partial \theta$ in Eq. (8) is not formidable. When $\varepsilon$ is specified as 0.005 , it is found that for the $1^{\text {st }}$ order sensitivities to the four shape variables $(\Lambda, \alpha, \tau$, and $A)$ the relative difference (averaged for the first 10 modes) between the finite difference and analytical approach is $0.003 \%, 0.003 \%, 0.002 \%$ and $0.003 \%$ respectively. The relative difference between the semi-analytical and analytical approach is $0.14 \%, 0.04 \%$, $0.02 \%$ and $0.01 \%$ respectively. Since the analytical approach gives the exact sensitivities, in this case the finite difference approach is more accurate than the semi-analytical one, however both the approaches yield quite accurate results.

For the original baseline wing, since the derivation of the analytical derivatives for ${ }^{\partial[G} 1 / \partial \theta$ is formidable, only the comparison of the $1^{\text {st }}$ order sensitivities using the finite difference and the semi-analytical approach was made. It is found in this case the sensitivities to the four shape variables using both the approaches are quite close, the average differences for the first 10 modes being in the range of $0.5 \sim 1.4 \%$. As an example, the $2^{\text {nd }}$ natural frequency w.r.t. $A$ is shown in Fig. $3(a)$, where it can be seen that the $1^{\text {st }}$ order sensitivities using the finite difference and the semi-analytical approaches almost coincide with each other. On the other hand, sensitivities to the sweep angle $\Lambda$ using the two approaches have had large relative differences especially for modes whose sensitivity to $\Lambda$ is small. One such example, the sensitivity of the $3^{\text {rd }}$ natural 
frequency w.r.t. $\Lambda$, is shown in Fig. 3(b). Here attention should be paid to the scale for the vertical coordinate to see how small the sensitivity to $\Lambda$ is.

It is observed in Fig. 3(b) that the finite difference approach has a better performance than the semianalytical one. In fact, in some extreme cases, the linear approximation using the first order sensitivity obtained using the semi-analytical approach is not at all tangent to the actual variation at the baseline point. This is not the case for the finite difference approach, if the step size chosen is not too large. Moreover, the computation efforts for both the approaches are at the same level since in both cases calculation of the stiffness and mass matrices at the baseline design and two perturbed designs should be performed.

It is obvious from observing Fig. 3 that the approximation using sensitivities up to the second order has much improved the results compared with the case where only the first order sensitivity is used. Also it can be seen that the second order sensitivities using the finite difference scheme of Eq. (4) are fairly accurate, at least for the purpose of engineering application. Since Eq. (4) shares the perturbation data with the first order sensitivity scheme, Eq. (3), therefore its evaluation requires little additional effort.

\section{Conclusion}

Modal response of general trapezoidal wing structures was investigated based on an equivalent model analysis and sensitivity techniques. The use of second order sensitivities proved to be yielding much better results than the case where only first order sensitivities are used. Shape sensitivities can be evaluated using analytical, finite difference and semi-analytical approaches. The present research shows that when the analytical solution is not available, the finite difference approach would be a better choice than the semianalytical one provided the step size is properly specified.

\section{Acknowledgments}

The support of NASA Langley Research Center on this research through Grant NAG-1-1884 with Drs. Jerry Housner and John Wang as the Technical Monitors is gratefully acknowledged. 


\section{References}

1. Karpel, M., Moulin, B. and Love, M. H., "Modal-Based Structural Optimization with Static Aeroelastic and Stress Constraints", Journal of Aircraft, Vol. 34, No. 3, 1997, pp. 433-440.

2. Haug, E. J., Choi, K. K. and Komkov, V., Design Sensitivity Analysis of Structural Systems, Academic Press, Inc., Orlando, Florida, 1986.

3. Adelman, H. M. and Haftka, R. T., "Sensitivity Analysis of Discrete Structural Systems", AIAA Journal, Vol. 24, No. 5, 1986, pp. 823-832.

4. Kapania, R. K., Bergen, F. D. and Barthelemy, J.-F. M., "Shape Sensitivity Analysis of Flutter Response of a Laminated Wing", AIAA Journal, Vol. 29, No. 4, 1991, pp. 611-612.

5. Singhvi, S. and Kapania, R. K., "Shape Sensitivities and Approximations of Modal Response of Laminated Skew Plates", Journal of Aircraft, Vol. 30, No. 3, 1993, pp. 423-426.

6. Eldred, L. B., "Sensitivity Analysis of the Static Aeroelastic Response of a Wing", Ph. D. Dissertation, Virginia Polytechnic Institute and State University, Feb. 1993

7. Kapania, R. K. and Issac, J. C., "Sensitivity Analysis of Aeroelastic Response of a Wing in Transonic Flow", AIAA Journal, Vol. 32, No. 2, 1994, pp. 350-356.

8. Bhardwaj, M. and Kapania, R. K., "Shape Sensitivity Analysis of Divergence Dynamic Pressure", Journal of Aircraft, Vol. 32, No. 4, 1995, pp. 898-901.

9. Kapania, R. K. and Liu, Y., "Static and Vibration Analyses of General Wing Structures Using Equivalent Plate Models", to appear in AIAA Journal. Also presented as Paper 2000-1434 at $4 l^{s t}$ AIAA/ASMEJASCE/AHS/ASC Structures, Structural Dynamics, and Materials Conference \& Exhibit, 3-6 April 2000, Atlanta, Georgia

10. Kapania, R. K. and Lovejoy, A. E., "Free Vibration of Thick Generally Laminated Cantilever Quadrilateral Plates”, AIAA Paper 95-1350, 1995, AIAA Journal, Vol. 34, No. 7, 1996, pp.1476-1486.

11. Cortial, F., "Sensitivity of Aeroelastic Response of Wings Using Equivalent Plate Models", Technical Report, Department of Aerospace and Ocean Engineering, Virginia Tech, 1996.

12. Rall, L. B., "Automatic Differentiation: Techniques and Applications", Lecture Notes in Computer Science, Vol. 120, Springer-Verlag, Berlin, 1981

13. Automatic Differentiation of Algorithms: Theory, Implementation, and Application, Ed. Griewank, A. and Corliss, G. F., SIAM, Philadelphia, 1992

14. Friswell, M. I. and Mottershead J. E., Finite Element Model Updating in Structural Dynamics, Kluwer Academic Publishers, the Netherlands, 1995. 


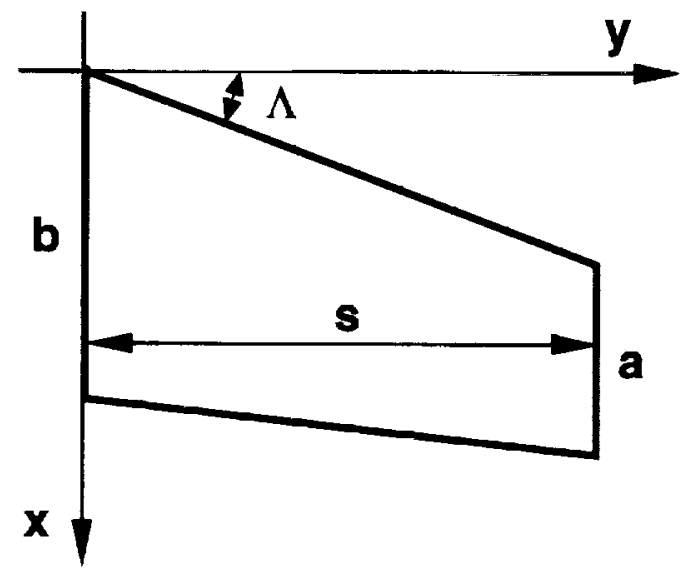

Fig. 1 Plan configuration of a trapezoidal wing

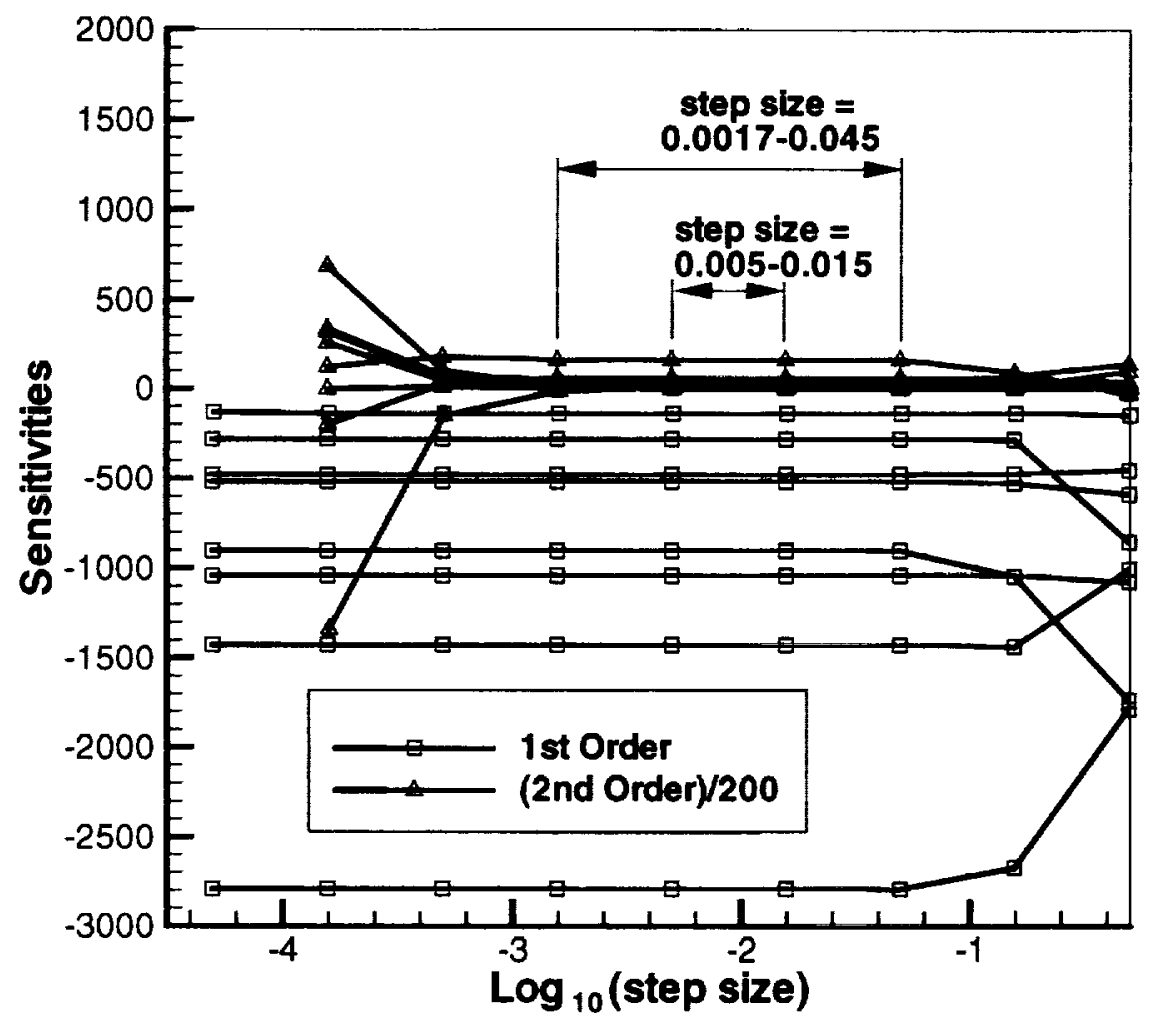

Fig. 2 Effect of the finite difference step size on the sensitivities 
of various natural frequencies to taper ratio
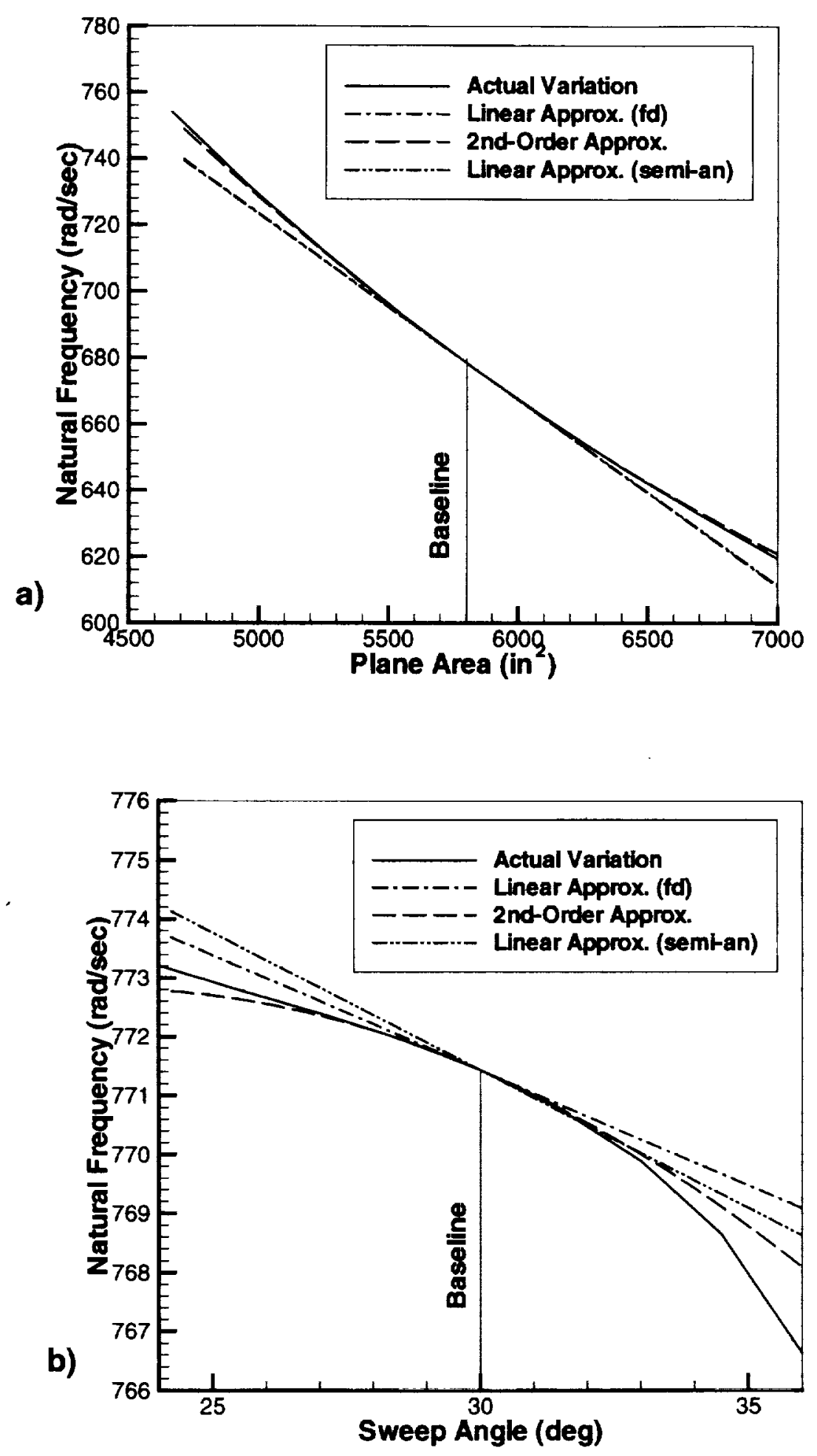

Fig. $31^{\text {st }}$ and $2^{\text {nd }}$ order sensitivities of a wing a) The $2^{\text {nd }}$ natural frequency w.r.t. wing plan area 
b) The $3^{\text {rd }}$ natural frequency w.r.t. wing sweep angle

Fig. 1 Plan configuration of a trapezoidal wing

Fig. 2 Effect of the finite difference step size on the sensitivities of various natural frequencies to taper ratio

Fig. $31^{\text {st }}$ and $2^{\text {nd }}$ order sensitivities of a wing

a) The $2^{\text {nd }}$ natural frequency w.r.t. wing plan area

b) The $3^{\text {rd }}$ natural frequency w.r.t. wing sweep angle 